\title{
Challenges, Open Research Issues and Tools in Bigdata Analytics
}

\author{
Rajesh Mothe, S Tharun Reddy, Kanegonda Ravi Chythanya,Y Supraja Reddy
}

\begin{abstract}
A large quantity of terabytes of data is produced each day from present day information structures and automated advances. For instance, Internet of Things and cloud computing. Analysis of these huge data takes a remarkable deal of endeavors at specific degrees to extricate understanding for primary leadership. In this way, large information evaluation is a go with the flow territory of revolutionary paintings. The essential goal of this paper is to explore the capability impact of large facts challenges, open research problems, and specific gadgets associated with it. Therefore, this text offers a stage to explore bigdata at various degrees. Also, it opens another skyline for specialists to execute the arrangement, in view of the demanding situations and open studies troubles.
\end{abstract}

Keywords-Big data analytics; Hadoop; MapReduce; Hadoop Distributed File System.

\section{INTRODUCTION}

In modernized days, information is created from various sources and the brisk advancement from cutting edge developments has incited improvement of enormous information. It outfits formative accomplishments in various fields with aggregation of huge datasets. At the point when all is said in done, it alludes to the social event of enormous and complex datasets which are difficult to process using ordinary database the official's gadgets or information planning applications. These are open in organized, semi-organized, and unstructured course of action in peta bytes and past. Formally, it is portrayed from $3 \mathrm{Vs}$ to $4 \mathrm{Vs}$. $3 \mathrm{Vs}$ alludes to volume, speed, and assortment. Volume alludes to the enormous measure of information that are being delivered customary however speed is the pace of improvement and how snappy the information is gathered for being examination. Assortment gives information about the sorts of information, for instance, organized, unstructured, semi organized, etc. The fourth $\mathrm{V}$ alludes to alludes that joins openness and duty. The essential objective of huge information investigation is to process information of high volume, speed, assortment, and alludes using particular standard and computational astute systems [1]. A segment of these extraction procedures for getting obliging information were discussed by Gandomi and Haider [2]. The accompanying Figure 1 alludes to the significance of bigdata. Anyway, cautious definition for bigdata isn't portrayed and

Revised Version Manuscript Received on 10 September, 2019.

Rajesh Mothe, Assistant Professor, Department of Computer Science and Engineering, SR Engineering College, Warangal, Telangana, India.

S Tharun Reddy, Assistant Professor, Department of Computer Science and Engineering, SR Engineering College, Warangal, Telangana, India.

Kanegonda Ravi Chythanya, Assistant Professor, Department of Computer Science and Engineering, SR Engineering College, Warangal, Telangana, India.

Y Supraja Reddy, Teaching Assistant, Department of Computer Science and Engineering, SR Engineering College, Warangal, Telangana, India.. there is an acknowledge that it is issue express.

It is every day that the improvement of big statistics is classed to arrive at 25 billion through 2015 [3]. From the perspective of the records and correspondence innovation, massive information is a hearty stimulus to the up and coming age of statistics innovation industries [4], which can be comprehensively created on the $1 / 3$ level, essentially alluding to bigdata, cloud computing, Internet of things, and social business. By and huge, Data distribution facilities had been utilized to deal with the large dataset. For this situation getting rid of the exact facts from the on hand huge facts is a first problem. The extra part of the exhibited methodologies in records mining aren't commonly ready to address the large datasets effectively. The key problem inside the analysis of bigdata is the absence of coordination among database structures just as with evaluation equipment, for instance, information mining and statistical evaluation. These demanding situations by way of and big emerge when we want to assemble information discovery and portrayal for its useful programs. A principal issue is the way through which to quantitatively depict the fundamental attributes of bigdata. There is a demand for epistemological ramifications in depicting facts disenchanted [5]. Moreover, the evaluation on intricacy speculation of big records will assist recognize fundamental attributes and development of complex examples in bigdata, enhance its portrayal, shows signs and symptoms of development understanding deliberation, and guide the plan of processing models and algorithms on bigdata [4]. Much research turned into achieved by means of special professionals on massive data and its patterns [6], [7], [8].

In any case, it's far to be noticed that each one facts gift as large statistics are not useful for analysis or simple leadership manner. Industry and the scholarly community are eager on scattering the discoveries of big statistics. This paper facilities round demanding situations in large data and its handy tactics. Moreover, we nation open research problems in big statistics. Thusly, to explain this, the paper is separated into following areas. Areas 2 preparations with challenges that emerge for the duration of adjusting of big facts. Area three clarify the open studies troubles with the intention to help us with processing bigdata and listen helpful expertise from it. Area four gives an expertise to large facts gear and techniques. End comments are given in vicinity 5 to define outcomes. 


\section{CHALLENGES IN BIG DATA ANALYTICS}

Ongoing year's huge data has been accrued in some areas like healthcare, public management, retail, bio chemistry, and other interdisciplinary logical seems into. Online programs experience huge records as often as possible, as an instance, social computing, internet textual content and documents, and net seek indexing. Social computing consists of informal business enterprise analysis, on-line networks, recommender systems, notoriety structures, and forecast markets wherein as internet seek index consists of ISI, IEEE Xplorer, Scopus, and Thomson Reuters and so on. Considering this focal factor of big statistics, it offers another open door inside the understanding getting ready assignments for the up and coming scientists. Anyway, openings continually pursue a few challenges. We talk approximately these problems quick in the accompanying subsections.

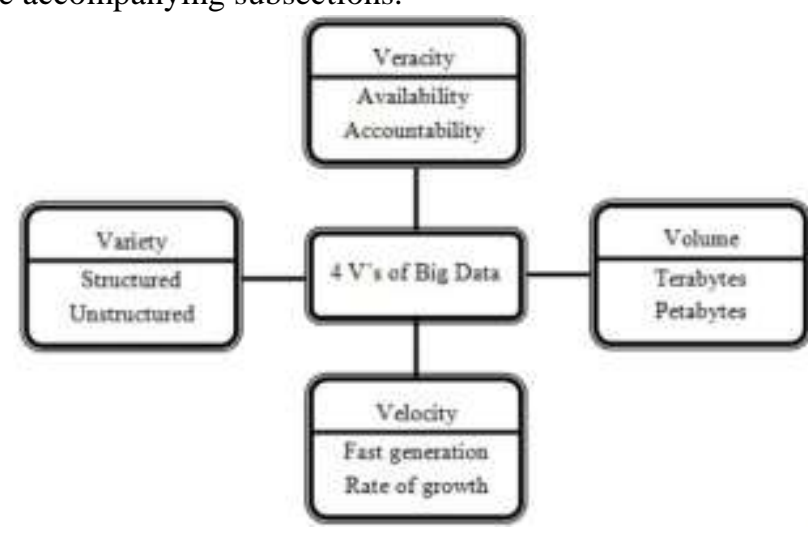

Fig. 1: Characteristics of Big Data

\section{A. Data Storage and Analysis}

As of past due the dimensions of records have evolved exponentially by means of exclusive strategies, for example, cellular phones, aeronautical tangible improvements, far off detecting, radio recurrence distinguishing proof peruses and so on. These statistics is put away on spending a lot fee though they left out or erased at long closing when you consider that there is no sufficient space to store them. In this manner, the principle check for bigdata analysis is capability mediums and better data/yield velocity. In such instances, the facts openness should be at the pinnacle want for the information discovery and portrayal. The prime reason is being that, it needs to be gotten to without difficulty and rapidly for in addition evaluation. In beyond a long time, investigator utilize tough disk drives to store information be that as it is able to, its extra sluggish abnormal data/yield execution than consecutive input/yield. To beat this obstacle, the concept of stable-nation pressure (SSD) and phrase trade memory (PCM) turned into provided. Anyway, the available garage advances can't have the desired exhibition for managing big data.

Another test with Big Data evaluation is ascribed to respectable kind of data. With the always developing of datasets, information mining assignments has essentially increased. Also, information reduction, facts choice, include willpower is an essential task mainly whilst handling huge datasets. This reveals an exceptional test for scientists. It is for the reason that, modern-day algorithms may not usually react in a first-rate time whilst coping with these high dimensional records. Mechanization of this gadget and growing new AI algorithms to ensure consistency is a noteworthy check recently. Notwithstanding these types of Clustering of big datasets that assist in studying the bigdata is of prime problem [10]. Late improvements, for example, Hadoop and MapReduce make it attainable to acquire large amount of semi structured and unstructured statistics in a realistic quantity of time [11]. The key constructing challenge is the way through which to appropriately break down this information for acquiring higher understanding. A trendy technique to this end is to trade the semi structed or unstructured data into structured facts, and after that follow facts mining algorithms to split information [12].

The giant takes a look at for this situation is to offer extra attention for planning storage structures and to raise effective information evaluation apparatus that give guarantees on the yield whilst the data originates from numerous resources. Moreover, plan of AI algorithms to explore information is fundamental for improving productiveness and flexibility.

\section{B. Knowledge Discovery and Computational Complexities}

Knowledge discovery and illustration is a top difficulty in massive records. It carries numerous sub fields, as an example, validation, submitting, the board, conservation, data healing, and portrayal. There are several devices for records discovery and portrayal, for example, fuzzy set [13], tough set [14], soft set [15], near set [16], formal idea analysis [17], precept element evaluation and so forth to provide a few examples. Furthermore, many hybridized techniques are moreover created to manner authentic problems. This kind of strategies are issue subordinate. Further a portion of those techniques may not be affordable for big datasets in a consecutive PC. Simultaneously a portion of the strategies have excellent attributes of adaptability over parallel PC. Since the dimensions of bigdata continues increasing exponentially, the handy units won't be gifted to process this information for obtaining crucial records. The maximum usual technique if there ought to get up a prevalence of massive dataset the executives is facts stockrooms and records stores. Data distribution middle is predominantly reliable to store records which are sourced from operational structures though information mart depends on an information stockroom and encourages analysis.

Analysis of large dataset requires progressively computational complexities. The serious trouble is to deal with irregularities and vulnerability gift inside the datasets. When all is stated in done, planned demonstrating of the computational multifaceted nature is utilized. It might be tough to set up an in-depth numerical gadget that is comprehensively applicable to Big Data. However, an area specific facts evaluation ought to be feasible efficiently by means of expertise the unique complexities. A development of such advancement ought to reproduce bigdata evaluation for numerous zones. Much research and examine has been performed closer to this direction utilizing AI strategies with the least reminiscence prerequisites. The essential intention in this exploration is to restriction computational fee dealing with and complexities [19], [20], [21]. 
In any case, modern bigdata evaluation gear have terrible showing in managing computational complexities, defenselessness, and abnormalities. It activates an exquisite take a look at to create strategies and improvements that can good buy computational intricacy, vulnerability, and irregularities in a feasible manner.

\section{C.Information Security}

In large records evaluation significant quantity of records are connected, analyzed, and dug for critical examples. All associations have numerous preparations to safe watchman their touchy records. Saving touchy statistics is a noteworthy trouble in massive records analysis. There is an enormous safety threat related with big records [22]. Along those traces, facts protection is becoming a chief statistics analysis difficulty. Security of huge records may be upgraded via using the systems of confirmation, approval, and encryption. Different protection efforts that big information applications face is length of gadget, extensive range of gadgets, non-stop protection checking, and absence of interruption gadget [23], [24]. The protection mission delivered about via bigdata has pulled inside the consideration of statistics security. In this manner, attention needs to take delivery of to accumulate a staggered security association version and anticipation device.

Albeit plenty research has been executed to confirm huge statistics yet it requires parcel of development. The good-sized check is to build up a staggered safety, protection saved information model for huge information.

\section{OPEN RESEARCH ISSUES IN BIG DATA ANALYTICS}

Big data evaluation and data technology are turning into the exploration point of convergence in ventures and the scholarly global. Data technological know-how targets investigating bigdata and expertise extraction from statistics. Uses of massive statistics and information science comprise statistics technology, vulnerability demonstrating, questionable statistics evaluation, AI, measurable knowledge, design acknowledgment, information warehousing, and sign preparing. Successful becoming a member of improvements and analysis will result in foreseeing the future drift of occasions. Principle focal factor of this area is to talk approximately open studies troubles in bigdata analysis. The exploration troubles regarding massive information analysis are arranged into 3 preferred classifications mainly Internet of things (IoT), cloud computing, bio propelled processing, and quantum computing. Anyway, it is not constrained to these issues.
More research problems diagnosed with fitness care large facts can be determined in Husing Kuo et al. Paper [9].

\section{A. IoT for Big Data Analytics}

Internet has rebuilt international interrelations, the craft of groups, social upsets and a mind-blowing quantity of person qualities. As of now, machines are becoming in on the demonstration to manipulate incalculable self-governing devices by way of internet and make Internet of Things (IoT). In this way, machines are becoming the patron of the internet, a good deal the same as human beings with the internet browsers. Internet of Things is drawing within the attention of overdue experts for its most encouraging chances and challenges. It has a fundamental financial and cultural impact for the destiny development of records, system and correspondence innovation. The new guideline of destiny might be in the end; the entirety may be related and shrewdly controlled. The idea of IoT is completing progressively suitable to the sensible global due to the advancement of cellular phones, installed and standard correspondence advances, cloud computing, and information evaluation. Also, IoT provides demanding situations in mixes of volume, pace and variety. In a greater considerable experience, a lot the same as the net, Internet of Things empowers the gadgets to exist in a horde of spots and encourages programs walking from minor to the important. Then once more, it's far as yet complicated to comprehend IoT nicely, such as definitions, substance and contrasts from other comparable thoughts. A few broadened improvements, for example, computational intelligence, and massive records can be consolidated together to improve the facts the board and understanding discovery of huge scale robotization applications. Much explore towards this route has been finished by using Mishra, Lin and Chang [25].

Data procurement from IoT data is the best test that bigdata expert is confronting. In this way, it's far primary to create foundation to examine the IoT records. An IoT system produces ceaseless floods of information and the analysts can create devices to extricate extensive information from this information making use of AI methods. Understanding these floods of statistics constituted of IoT gadgets and breaking down them to get essential facts is a tough issue and it prompts bigdata evaluation. AI algorithms and computational know-how strategies is the primary solution for cope with large information from IoT planned. Key innovations that are related with IoT are additionally talked about in many studies papers [26]. Figure 2 delineates an outline of IoT bigdata and information discovery process. 


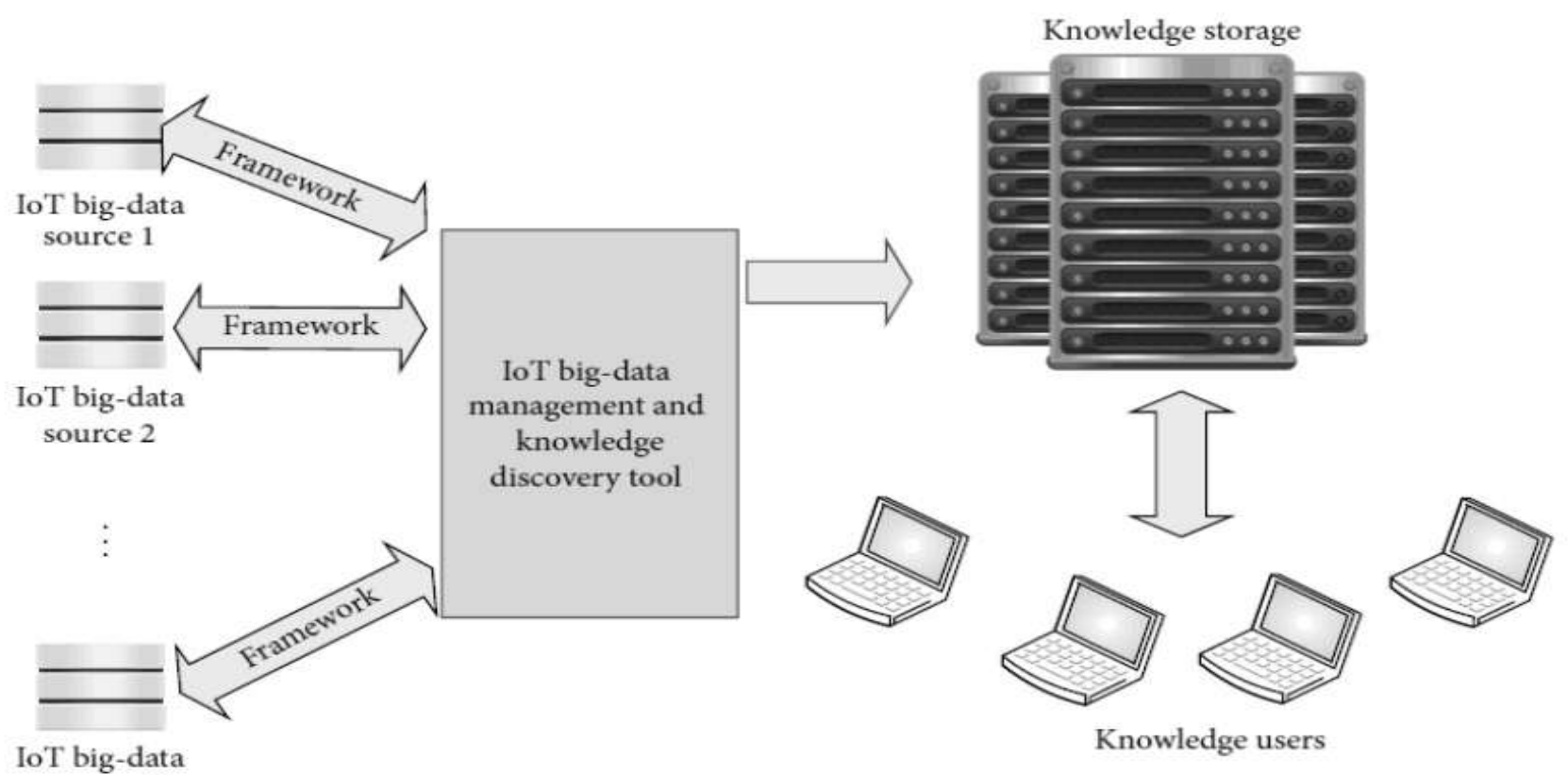

Fig. 2: IoT Big Data Knowledge Discovery

Knowledge analysis device has begun from hypotheses of human records making ready, for example, outlines, guidelines, labeling, and semantic structures. As a rule, it comprises of four fragments, as an example, knowledge securing, data base, understanding spread, and information utility. In facts procurement degree, know-how is discovered by using utilizing one of a kind conventional and computational know-how strategies. The determined knowledge is put away in facts bases and master systems are through and massive dependent on the observed information. Knowledge scattering is widespread for getting critical facts from the information base. Knowledge extraction is a process that searches documents, data internal statistics simply as expertise bases. The closing level is to apply determined information in one-of-a-kind programs. It is a definitive objective of statistics disclosure. The data analysis device is essentially iterative with the judgment of expertise application. There are numerous issues, exchanges, and examines in this issue remember analysis. It is beyond volume of this examine paper. For better representation, expertise evaluation system is delineated in Figure three.

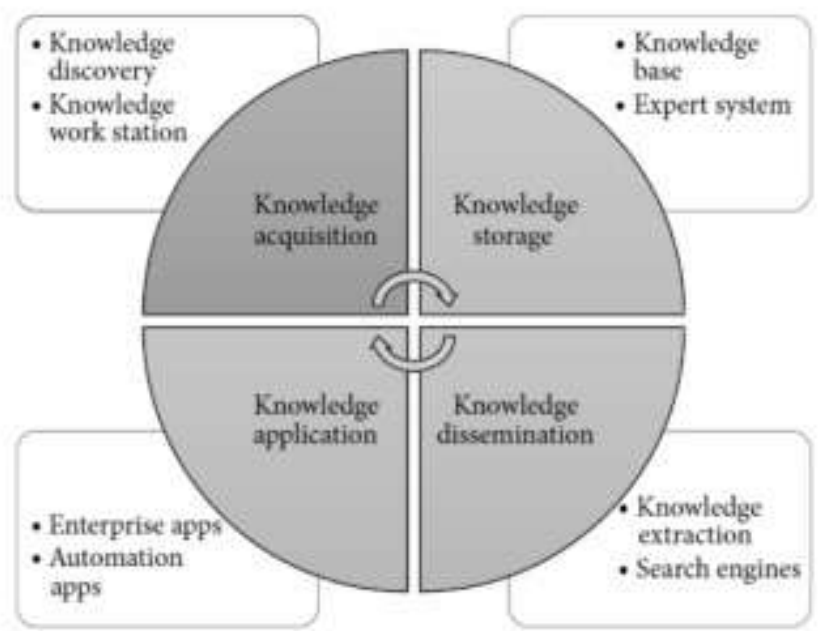

Fig. 3: IoT Knowledge Exploration System

\section{B. Cloud Computing for Big Data Analytics}

The development of virtualization advances has made supercomputing steadily available and moderate. Registering structures which can be covered up in virtualization programming make systems to hold on like an actual PC, but with the adaptability of particular subtleties, as an example, wide variety of processors, plate area, memory, and working system. The utilization of those digital PCs is referred to as dispensed computing which has been one of the heartiest bigdata methods. Large Data and allotted computing innovations are created with the importance of constructing up an adaptable and on interest accessibility of belongings and information. Distributed computing fit bigdata with the aid of on hobby

Access to configurable registering belongings thru virtualization approaches. The blessings of the use of the Cloud processing include supplying assets while there's a hobby and pay only for the belongings that's predicted to accumulate the item. All the while, it improves accessibility and value decrease. Open challenges and research issues of large information and allotted computing are pointed out in detail by means of several professionals which features the challenges in records the board, information variety and pace, facts storage, statistics managing, and asset the executives [27], [28]. So, Cloud processing enables in constructing up a course of action for all types of uses with basis and instruments.

Big statistics software making use of distributed computing should bolster facts logical and development. The cloud condition ought to provide devices that permit data researchers and enterprise professionals to intelligently and cooperatively discover expertise acquiring records for in addition handling and doing away with efficient effects. This can fathom large applications that could emerge in one-of-a-kind regions. Moreover, disbursed computing need

\section{Published By:}

Blue Eyes Intelligence Engineering 
to likewise empower scaling of tools from digital advances into new improvements like flash, $\mathrm{R}$, and extraordinary kinds of bigdata preparing strategies.

Big information shapes a shape for analyzing allotted computing alternatives. Contingent upon particular need, client can visit the industrial center and buy machine administrations from cloud professional co-ops, for example, Google, Amazon, IBM, and programming as an administration (SaaS) from a whole crew of corporations, as an example, NetSuite, Cloud9, and Jobscience and so on. Another bit of leeway of allotted computing is sent storage which offers a manageable method to putting away bigdata. The undeniable one is the time and value which might be predicted to transfer and download bigdata inside the cloud situation. Else, it winds up difficult to control the dissemination of calculation and the hidden equipment. Be that as it could, the severe issues are protection issues identifying with the facilitating of statistics on open servers, and the potential of statistics from human analysis. This type of problems will take bigdata and dispensed computing to an abnormal kingdom of development.

\section{Bio-inspired Computing for Big Data Analytics}

Bio-roused processing is a technique propelled normally to deal with complicated actual troubles. Natural structures are self-composed without a focal manage. A bio-enlivened cost minimization tool search and find the right data administration arrangement on thinking about cost of facts the executives and management help. These strategies are created by using natural debris, for example, DNA and proteins to direct computational counts inclusive of placing away, getting better, and getting ready of facts. A massive element of such registering is that it coordinates organically inferred substances to perform computational capacities and get keen execution. These systems are gradually reasonable for big statistics programs. Colossal amount of statistics is created from type of belongings over the net since the digitization. Examining these statistics and ordering into content, photograph and video and so on would require parcel of smart evaluation from statistics researchers and large records specialists. Multiplications of innovations are growing like bigdata, IoT, dispensed computing, bio enlivened registering and so forth though balance of records should be possible simply by choosing right stage to investigate massive and outfit realistic consequences.

\section{TOOLS FOR BIG DATA PROCESSING \& RESULTS}

Big quantities of devices are handy to system bigdata. In this segment, we observe a few gift strategies for investigating huge statistics with accentuation on 3 widespread developing gadgets specifically MapReduce, Apache Spark, and Storm. The substantial majority of the available gadget's attention on clump handling, flow getting ready, and intuitive evaluation. Most group managing devices depend on the Apache Hadoop basis, as an example, Mahout and Dryad. Stream records applications are for the most element applied for ongoing investigative. A few instances of huge-scale spilling degree are Strom and Splunk. The intuitive analysis procedure enables clients to legitimately collaborate continuously for his or her very personal analysis. For instance, Dremel and Apache Drill are the big statistics levels that help wise evaluation. These gadgets assist us in building up the bigdata ventures. A remarkable rundown of large facts devices and tactics is moreover mentioned by way of numerous experts [6], [29]. The regular paintings movement of huge information undertaking pointed out by Huang et al is featured on this place and is delineated in Figure 4.

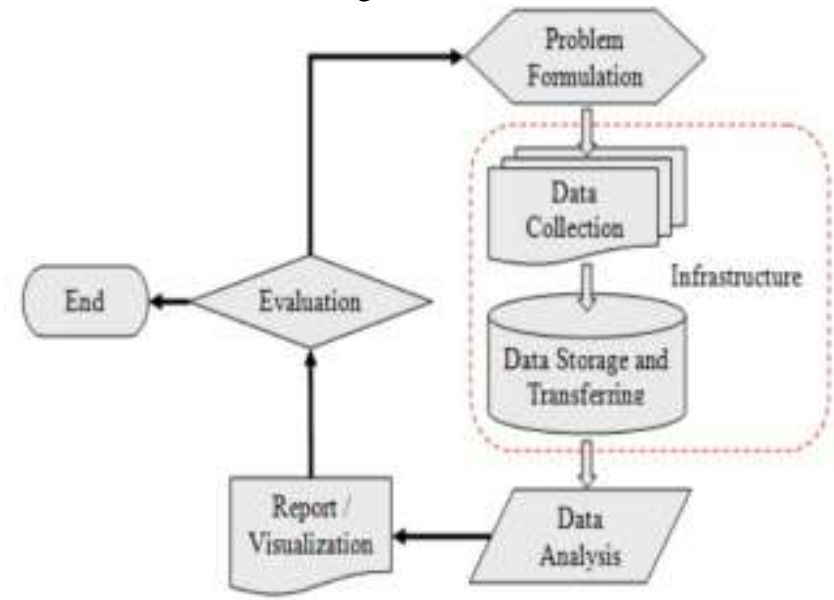

Fig. 4: Workflow of Big Data Project

\section{A. Apache Hadoop and MapReduce}

The maximum settled programming degree for big records evaluation is Apache Hadoop and MapReduce. It incorporates of Hadoop component, MapReduce, Hadoop distributed file system (HDFS) and apache hive and so on. Guide diminish is a programming version for getting ready huge datasets relies upon on hole and conquer method. The partition and conquer approach are actualized in tiers, for instance, Map step and Reduce Step. Hadoop takes a shot at two sorts of hubs, as an example, ace hub and laborer hub. The ace hub separates the contribution to littler sub issues and later on disperses them to professional hubs in manual step. From that point the ace hub consolidates the yields for all the sub troubles in diminish step. In addition, Hadoop and MapReduce fill in as a great programming shape for looking after massive statistics issues. It is additionally beneficial in deficiency tolerant potential and excessive throughput records coping with.

\section{B. Apache Mahout}

Apache mahout intends to give flexible and business AI techniques for big scale and intelligent statistics evaluation applications. Center algorithms of mahout which include clustering, grouping, layout mining, relapse, dimensionality lower, transformative algorithms, and clump prepare collective isolating hold walking with respect to top of Hadoop level thru guide lessen shape. The objective of mahout is to fabricate an active, responsive, diverse network to inspire talks on the project and capability use instances. The fundamental goal of Apache mahout is to offer an apparatus to lifting massive challenges. The various groups the individuals who've actualized adaptable AI algorithms are Google, IBM, Amazon, Yahoo, Twitter, and Facebook [31]. 


\section{Apache Spark}

Apache Spark is an open source bigdata making ready device worked for pace managing, and complex evaluation. It is whatever but tough to utilize and changed into to begin with created in 2009 in UC Berkeley's AMP Lab. It became publicly released in 2010 as an Apache venture. Flash lets you hastily compose programs in java, Scala, or python. Notwithstanding manual decrease duties, it underpins SQL inquiries, spilling information, AI, and diagram facts managing. Flash continues running over existing Hadoop distributed report device (HDFS) basis to provide upgraded and additional usefulness. Sparkle comprises of segments in particular motive force software, cluster chief and professional hubs. The driving force program fills in as the start degree of execution of a utility on the flicker group. The cluster administrator distributes the property and the professional hubs to do the statistics preparing as errands. Every software can have plenty of methods considered sellers that are in price of executing the assignments. The actual bit of leeway is that it gives assist for sending flash programs in a modern-day Hadoop cluster. Figure 5 portrays the design chart of Apache Spark.

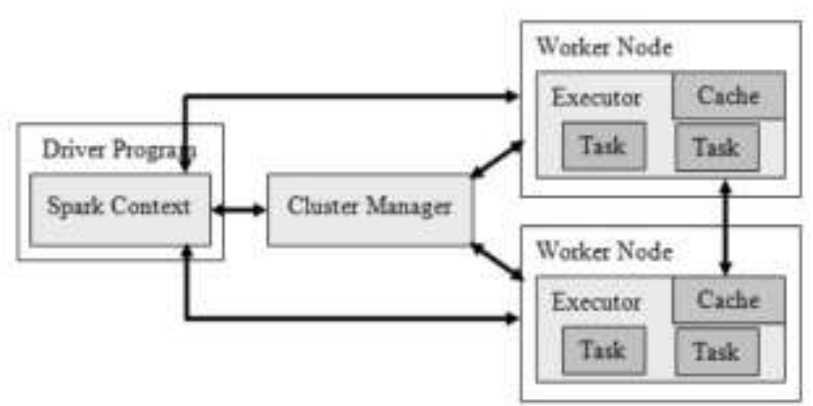

Fig. 5: Architecture of Apache Spark

\section{Dryad}

It is any other outstanding programming version for executing parallel and appropriated packages for looking after large setting bases on dataflow chart. It contains of a cluster of registering hubs, and a purchaser make use of the assets of a PC institution to run their application in a circulated manner. Without a doubt, a dryad purchaser utilizes a big wide variety of machines, each one of them with several processors or centers. The large little bit of leeway is that customers don't ought to know something about simultaneous programming. A dryad software runs a computational coordinated diagram this is created from computational vertices and correspondence channels. Accordingly, dryad gives an big variety of usefulness which includes developing of employment diagram, reserving of the machines for the handy approaches, change unhappiness handling in the cluster, gathering of execution anointments, picturing the interest, summoning patron characterized methods and powerfully fresh the pastime chart in light of those association selections without knowing the semantics of the vertices [32].

\section{E. Storm}

Tempest is a conveyed and deficiency tolerant consistent calculation system for making ready big gushing statistics. It is relatively intended for continuous handling in sticks out from Hadoop that's for cluster getting ready. Furthermore, it's miles additionally simple to set up and paintings, versatile, flaw tolerant to present centered exhibitions. The tempest group is evidently like Hadoop cluster. On tempest group customers run numerous topologies for various tempest undertakings whilst Hadoop stage actualizes guide decrease occupations for evaluating packages. There is quantity of contrasts between manual lessen employments and topologies. The fundamental assessment is that guide decrease work ultimately completes at the same time as a topology techniques messages continuously, or until client give up it. A tempest cluster contains of sorts of hubs, for example, ace hub and professional hub. The ace hub and expert hub execute styles of jobs, for instance, glow and supervisor for my part. The jobs have comparative capacities as per task tracker and task tracker of guide lessen machine. Aura is chargeable for conveying code over the tempest cluster, booking and appointing undertakings to laborer hubs, and checking the entire gadget. The boss goes alongside errands as doled out to them with the aid of radiance. Moreover, it begins and ends the procedure as important dependent on the guidelines of air of secrecy. The entire computational innovation is apportioned and conveyed to numerous laborer bureaucracy and every specialist manner actualizes a chunk of the topology.

\section{F. Splunk}

Lately a top-notch deal of records is created thru machine from business enterprises. Splunk is a continuous and keen level created for abusing gadget produced big information. It joins the up to the moment cloud advances and bigdata. Thusly it encourages patron to look, display screen, and examine their machine created information via net interface. The effects are shown in an instinctive way, for instance, diagrams, reviews, and cautions. Splunk is particular in terms of other stream dealing with gear. Its quirks comprise ordering organized, unstructured device produced records, steady searching, detailing diagnostic effects, and dashboards. The maximum tremendous intention of Splunk is to provide anointments to several packages, analyze issues for machine and records innovation foundations, and canny help for commercial enterprise sports.

\section{SUGGESTIONS FOR FUTURE WORK}

The quantity of statistics collected from exceptional programs everywhere for the duration of internationally an extensive form of fields today is relied upon to twofold at normal durations. It has no utility besides if these are tested to get precious information. This calls for the advancement of structures which can be utilized to encourage huge records evaluation. The development of notable PCs is a help to execute these techniques prompting automatic structures. The exchange of facts into expertise is in no way, form or shape an easy task for elite large-scale information preparing, which includes misusing parallelism of present and up and coming PC systems for records mining. Besides, these statistics may additionally include vulnerability in an 
extensive range of systems. An extensive variety of models like fluffy units, unsightly sets, delicate sets, neural structures, their speculations and half of-breed fashions gotten by way of joining as a minimum of those models were found to be efficient in speak me to records. These models are likewise particularly efficient for evaluation. As a preferred rule, bigdata is dwindled to include simply the vast attributes vital from a selected report angle or relying on the utility place. Along those lines, decrease structures were created. Regularly the facts gathered have missing traits. These traits ought to be created or the tuples having these missing characteristics are disbursed with from the detail series earlier than analysis. All the more significantly, these new demanding situations may additionally encompass, every so often even smash down, the exhibition, scalability and scalability of the devoted statistics escalated processing systems. The later method now and then prompts lack of data and henceforth no longer appreciated. This raises many research issues in the commercial enterprise and studies community in styles of catching and attending to facts successfully. Also, brief coping with even as at the identical time conducting advanced and high throughput, and putting away it productively for a while later is any other trouble. Further, programming for bigdata evaluation is a huge testing trouble. Communicating facts get to prerequisites of utilizations and structuring programming language reflections to abuse parallelism are a quick need [33].

Moreover, AI ideas and tools are choosing up reputation amongst professionals to encourage crucial effects from those ideas. Research inside the vicinity of AI for bigdata has focused on statistics managing, calculation utilization, and streamlining. A big wide variety of the AI tools for bigdata are started as of overdue desires unusual trade to get hold of it. We contend that even as each one of the contraptions has their points of interest and impediments, more and more effective devices may be produced for coping with problems intrinsic to bigdata. The powerful contraptions to be created have to have arrangement to address uproarious and irregularity facts, vulnerability and irregularity, and lacking qualities.

\section{CONCLUSION}

As of overdue statistics are created at a dramatic tempo. Examining these statistics is making an attempt for a popular guy. To this led to this paper, we evaluate the one-of-a-kind research troubles, demanding situations, and gear used to research this bigdata. From this examine, it's far comprehended that every bigdata stage has its character center interest. Some of them are meant for cluster coping with although some are wonderful at constant expository. Each large data stage moreover has explicit usefulness. Various strategies utilized for the evaluation incorporate statistical analysis, AI, data mining, eager evaluation, distributed computing, quantum registering, and statistics circulate making ready. We be given that in future experts will give greater consideration to these techniques to attend to issues of bigdata viably and proficiently.

\section{REFERENCES}

1. M. K.Kakhani, S. Kakhani and S. R.Biradar, Research issues in big data analytics, International Journal of Application or Innovation in Engineering \& Management, 2(8) (2015), pp.228-232.

2. A. Gandomi and M. Haider, Beyond the hype: Big data concepts, methods, and analytics, International Journal of Data Management, 35(2) (2015), pp.137-144.

3. C. Lynch, Big data: How do your data grow? Nature, 455 (2008), pp.28-29.

4. X. Jin, B. W.Wah, X. Cheng and Y. Wang, Significance and challenges of big data research, Big Data Research, 2(2) (2015), pp.59-64.

5. R. Kitchin, Big Data, new epistemologies and paradigm shifts, Big Data Society, 1(1) (2014), pp.1-12.

6. C. L. Philip, Q. Chen and C. Y. Zhang, Data-intensive applications, challenges, techniques and technologies: A survey on big data, Data Sciences, 275 (2014), pp.314-347.

7. M. Rajesh, P.Kumara Swamy, Secure Message Authentication in Pervasive Computing, International Journal for Technological Research in Engineering, Vol. 3-4, DEC 2015, Page No. 840-843, ISSN/ISBN No. 2347 $-4718$.

8. S. Del. Rio, V. Lopez, J. M. Bentez and F. Herrera, On the use of mapreduce for imbalanced big data using random forest, Data Sciences, 285 (2014), pp.112-137.

9. MH. Kuo, T. Sahama, A. W. Kushniruk, E. M. Borycki and D. K. Grunwell, Health big data analytics: current perspectives, challenges and potential solutions, International Journal of Big Data Intelligence, 1 (2014), pp.114-126.

10. Z. Huang, A fast clustering algorithm to cluster very large categorical data sets in data mining, SIGMOD Workmart on Research Issues on Data Mining and Knowledge Discovery, 1997.

11. T. K. Das and P. M. Kumar, Big data analytics: A system for unstructured data analysis, International Journal of Engineering and Technology, 5(1) (2013), pp.153-156.

12. T. K. Das, D. P. Acharjya and M. R. Patra, Opinion mining about a product by analyzing public tweets in twitter, International Conference on Computer Communication and Informatics, 2014

13. L. A. Zadeh, Fuzzy sets, Data and Control, 8 (1965), pp.338-353

14. Z. Pawlak, Rough sets, International Journal of Computer Data Science, 11 (1982), pp.341-356.

15. D. Molodtsov, Soft set theory first results, Computers and Mathematics with Aplications, 37(4/5) (1999), pp.19-31.

16. J. F.Peters, Near sets. General theory about nearness of objects, Applied Mathematical Sciences, 1(53) (2007), pp.2609-2629.

17. M. Rajesh, B. Swathi, Y. Chanti, B. Vijay Kumar, A Modified Fault Diagnosis Scheme in Wireless Sensor Networks, International Journal of Engineering Technology, Vol. 7-8, DEC 2018, Page No. 226-229, ISSN/ISBN No. 2227-524X.

18. I. T.Jolliffe, Principal Component Analysis, Springer, New York, 2002.

19. O. Y. Al-Jarrah, P. D. Yoo, S. Muhaidat, G. K Karagiannidis and K. Taha, Efficient machine knowledge for big data: A review, Big Data Research, 2(3) (2015), pp.87-93. 
20. Changwon. Y, Luis. Ramirez and Juan. Liuzzi, Big data analysis using modern statistical and machine knowledge methods in medicine, International Neurourology Journal, 18 (2014), pp.50-57.

21. P. Singh and B. Suri, Quality assessment of data using statistical and machine knowledge methods. L. C.Jain, H. S.Behera, J. K.Mandal and D. P.Mohapatra (eds.), Computational Intelligence in Data Mining, 2 (2014), pp. 89-97.

22. H. Zhu, Z. Xu and Y. Huang, Research on the security technology of big data data, International Conference on Data Technology and Management Innovation, 2015, pp.1041-1044.

23. Z. Hongjun, H. Wenning, H. Dengchao and M. Yuxing, Survey of research on data security in big data, Congresso da sociedada Brasileira de Computacao, 2014, pp.1-6.

24. I. Merelli, H. Perez-sanchez, S. Gesing and D. D.Agostino, Managing, analysing, and integrating big data in medical bioinformatics: open problems and future perspectives, BioMed Research International, 2014,(2014), pp.1-13.

25. N. Mishra, C. Lin and H. Chang, A cognitive adopted system for iot big data management and knowledge discovery prospective, International Journal of Distributed Sensor Networks, 2015, (2015), pp.1-13

26. X. Y.Chen and Z. G.Jin, Research on key technology and applications for internet of things, Physics Procedia, 33, (2012), pp. 561-566.

27. M. Rajesh, B. Vijay Kumar, Sunil, Big Data Technology Applications in Automated Health Care Management System, International Journal of Advanced Research in Computer Science, Vol. 8-9, DEC 2017, Page No. 115-120, ISSN/ISBN No. 0976-5697.

28. I. A. T. Hashem, I. Yaqoob, N. Badrul Anuar, S. Mokhtar, A. Gani and S. Ullah Khan, The rise of big data on cloud computing: Review and open research issues, Data Systems, 47 (2014), pp. 98-115.

29. M. Herland, T. M. Khoshgoftaar and R. Wald, A review of data mining using big data in health informatics, Journal of Big Data, 1(2) (2014), pp. 1-35.

30. T. Huang, L. Lan, X. Fang, P. An, J. Min and F. WangPromises and challenges of big data computing in health sciences, Big Data Research, 2(1) (2015), pp. 2-11.

31. G. Ingersoll, Introducing apache mahout: Scalable, commercial friendly machine knowledge for building intelligent applications, White Paper, IBM Developer Works, (2009), pp. 1-18.

32. H. Li, G. Fox and J. Qiu, Performance model for parallel matrix multiplication with dryad: Dataflow graph runtime, Second International Conference on Cloud and Green Computing, 2012, pp.675-683.

33. D. P. Acharjya, S. Dehuri and S. Sanyal Computational Intelligence for Big Data Analysis, Springer International Publishing AG, Switzerland, USA, ISBN 978-3-319-16597-4, 2015. 